\title{
Optimum signal re-processing of multiple legacy surveys to improve pre-salt imaging in the complex Santos Basin, Brazil
}

Hongyan Li, Oscar Pérez, Carina Lansky, Basim Shalab, and Teddy L. Standley, Schlumberger.

Copyright 2021, SBGf - Sociedade Brasileira de Geofísica

This paper was prepared for presentation during the $17^{\text {th }}$ International Congress of the Brazilian Geophysical Society held in Rio de Janeiro, Brazil, 16-19 August 2021.

Contents of this paper were reviewed by the Technical Committee of the $17^{\text {th }}$ International Congress of the Brazilian Geophysical Society and do not necessarily represent any position of the SBGf, its officers or members. Electronic reproduction or storage of any part of this paper for commercial purposes without the written consent of the Brazilian Geophysical Society is prohibited.

\section{Abstract}

Santos Basin is emerging as one of the world's most promising exploration and production areas. The pre-salt region is complex, with fast carbonates, slow sediments, volcanic layers, and large fault systems. Together with the complicated overburden structures including carbonate layers in the post salt and stratified salt, high-resolution imaging is critical to better understand the producing fields and to facilitate development of others. High-resolution imaging relies on adequate illumination, seismic data with sufficient resolution and a good signal-to-noise (SNR), and an accurate velocity model. The accuracy of the velocity model is also dependent on the quality of seismic data. In the central Santos Basin, by combining several legacy surveys along different shooting directions, we show that the illumination of the target zones is increased. With signal processing technologies developed in recent years, we show that the SNR and resolution of the data were improved. Surface multiple attenuation is challenging in this area due to the existence of high-velocity, steepdipping post-salt carbonates and stratified evaporites. We show that multiple prediction is improved by the larger crossline aperture enabled by the incorporation of all the legacy data; therefore, improved multiple elimination was achieved. With the reprocessed higher resolution and higher SNR data and increased azimuth sampling, a more accurate velocity model is inverted by contemporary model building technologies. Finally, delivering high-resolution presalt imaging.

\section{Introduction}

In the central Santos Basin, several legacy surveys are available, acquired between 2002 to 2011 with various acquisition configurations (Figure 1; Table 1). There are three narrow-azimuth (NAZ) data with acquisition azimuths $\sim 34^{\circ}$ apart. Additionally, one survey acquired with a circular shooting technique covers a small portion of the area with full-azimuth (FAZ) data coverage. With the developments of signal processing and depth imaging in recent years, the central Santos multi-azimuth (MAZ) reimaging project was initiated at the end of 2019. Ortin et al. (2020) provided a comprehensive introduction to the reprocessing effort. The results from the fast-track processing show significant improvements in the pre-salt imaging against the legacy result (Ortin et al., 2020), obtained by adaptive deghosting (Rickett, 2014; Zarkhidze et al., 2016), velocity updates inside the salt and pre-salt zone, and the image combination of one of the NAZ surveys and the FAZ survey.

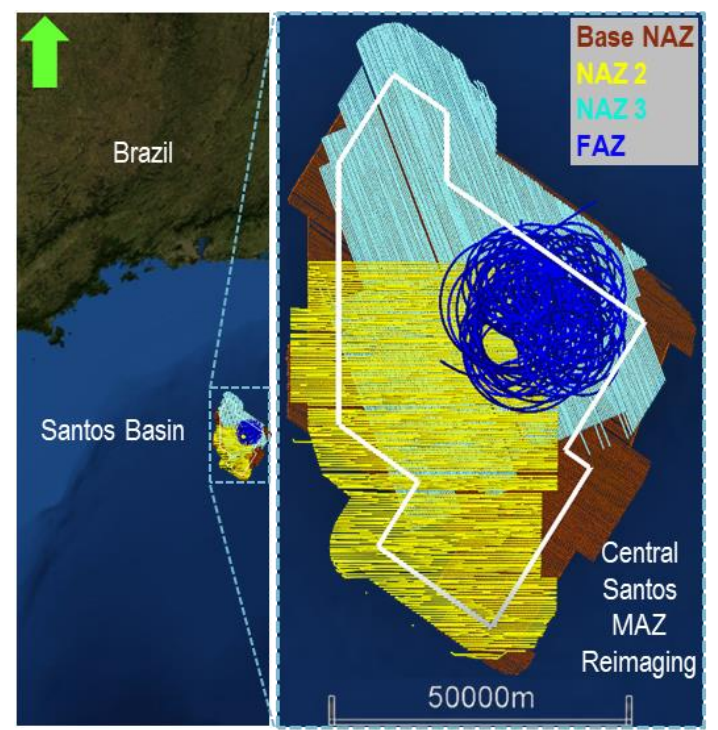

Figure 1 - Central Santos MAZ Reimaging project area and input shot maps from all the surveys.

In this study, a contemporary signal processing flow (Figure 2) is applied consistently to the three NAZ and the FAZ legacy datasets. With several data examples we provide a deeper insight on the key methodologies and strategies that have been used to generate the improved seismic image.

We focus on three main aspects:

1. Increasing the signal-to-noise ratio(SNR): the legacy data had relatively shallow tow depths, as was typical for the years when they were acquired, and so exhibited a significant amount of swell noise.

2. Extending the useful bandwidth in the data spectrum on both low and high end: With the first ghost notch being at $0 \mathrm{~Hz}$, most of the low frequencies are suppressed. The next notch in the higher frequencies also reduces seismic resolution.

3. Combining all the available data to generate a robust surface multiple model: previously processed data showed strong residual multiple energy in some places, which compromised the quality of the final image. Meanwhile, multiples complicate the picking of primary events during seismic tomography especially in the intra-salt and pre-salt 
zones, and they also challenge the interpretation of structures and faults.

\begin{tabular}{|c|c|c|c|c|}
\hline Parameter & Base NAZ & NAZ 2 & NAZ 3 & FAZ \\
\hline Acquisition grid & $6.25 \times 12.5 \mathrm{~m}$ & $6.25 \times 12.5 \mathrm{~m}$ & $6.25 \times 37.5 \mathrm{~m}$ & $25.0 \times 25.0 \mathrm{~m}$ \\
\hline Number of cables & 12 & 14 & 6 & 10 \\
\hline Cable separation & $50 \mathrm{~m}$ & $50 \mathrm{~m}$ & $150 \mathrm{~m}$ & $120 \mathrm{~m}$ \\
\hline Cable length & $8000 \mathrm{~m}$ & $8100 \mathrm{~m}$ & $6000 \mathrm{~m}$ & $8000 \mathrm{~m}$ \\
\hline Cable depth & $9 \mathrm{~m}$ & $9 \mathrm{~m}$ & $9 \mathrm{~m}$ & $12 \mathrm{~m}$ \\
\hline Number of sources & \multicolumn{5}{|c|}{2} \\
\hline Source separation & $25 \mathrm{~m}$ & $25 \mathrm{~m}$ & $75 \mathrm{~m}$ & $60 \mathrm{~m}$ \\
\hline Source depth & $7 \mathrm{~m}$ & $7 \mathrm{~m}$ & $7 \mathrm{~m}$ & $10 \mathrm{~m}$ \\
\hline Shotpoint interval & $25 \mathrm{~m}$ (flip-flop) & $25 \mathrm{~m}$ (flip-flop) & $25 \mathrm{~m}$ (flip-flop) & $37.5 \mathrm{~m}$ (flip-flop) \\
\hline Group interval & \multicolumn{5}{|c|}{$12.5 \mathrm{~m}$} \\
\hline Azimuth & $123.6 / 303.6$ & $158 / 338$ & $90 / 270$ & \\
\hline
\end{tabular}

Table 1 - Input survey parameters.

\section{Method}

To increase the SNR, a 3D adaptive noise attenuation workflow (ADNA) is applied to the data. ADNA models the low frequency random noise that is inconsistent with the physics of plane wave propagation, and a curvelet based subtraction ( $\mathrm{Yu}$ and $\mathrm{Yan}, 2011)$ ensured an effective attenuation of the noise while preserving the signal. Linear noise, spikes and seismic interference are addressed in subsequent residual de-noise steps. Figure 3 compares one shot before/after ADNA and residual noise attenuation and filtered to $10 \mathrm{~Hz}$. The strong swell noise is effectively attenuated without any signal leakage. The attenuation of the strong low-frequency noise is significant for the success of full-waveform inversion (Vigh and Starr, 2008) to update the velocity model.

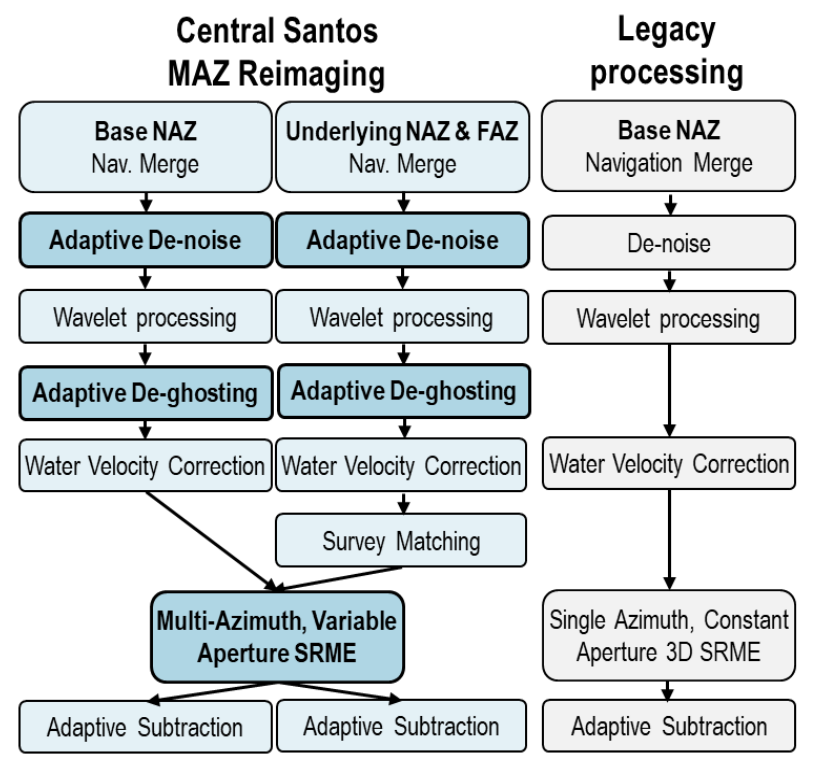

Figure 2 - Central Santos MAZ Reimaging pre-processing sequence and the 2015 legacy pre-processing sequence as reference.
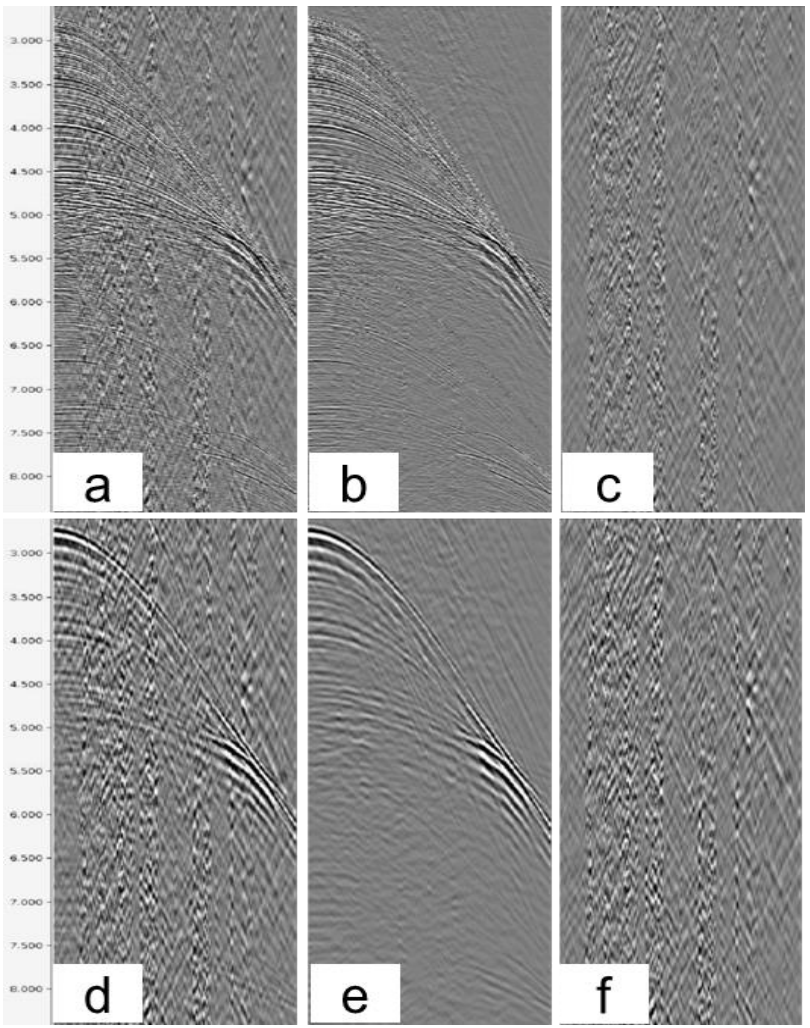

Figure 3 - (a). Raw shot; (b). After ADNA and residual noise attenuation; (c). The difference of (a) and (b). (d)-(f) are band limited displays of (a)-(c), filtered to a maximum frequency of $10 \mathrm{~Hz}$.

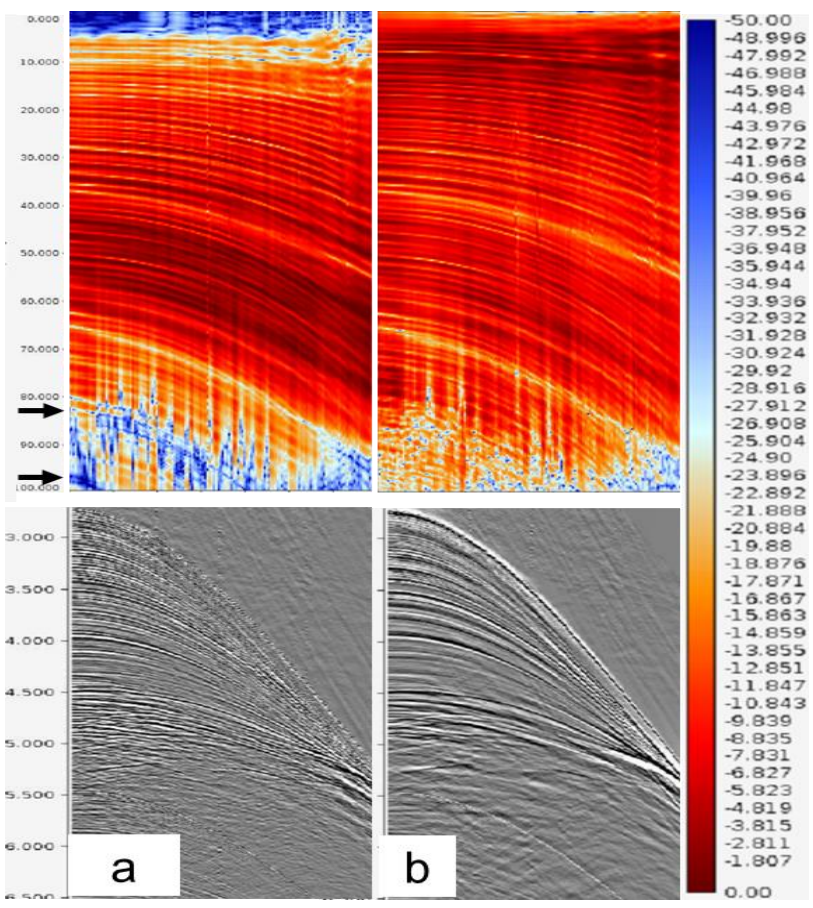

Figure 4 - F-X plot (top) of one shot (bottom) before (a) and after (b) $A D$. 
Following attenuation of the noise, adaptive deghosting was applied to simultaneously eliminate source and receiver ghosts, therefore, providing high-resolution broadband data. The F-X plot in Figure 4 compares one shot before and after deghosting. The two arrows point to the high-frequency notch related to the source and receiver ghost. After deghosting, the two notches are removed, and the low frequencies between $0-10 \mathrm{~Hz}$ are very well recovered, which is critical for steep-dipping events and presalt imaging.

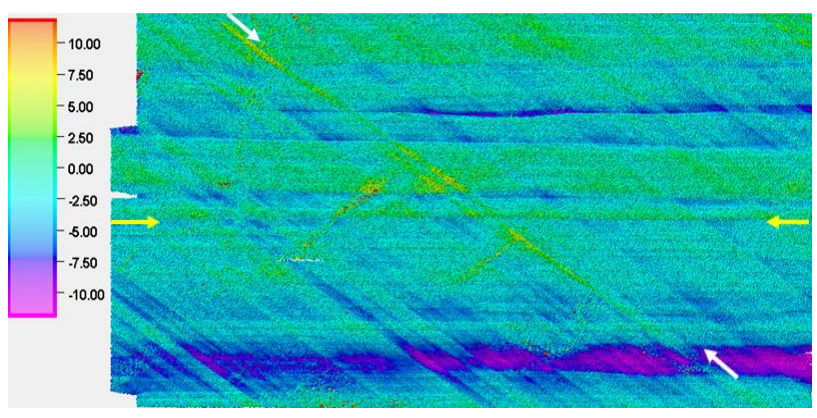

(a)

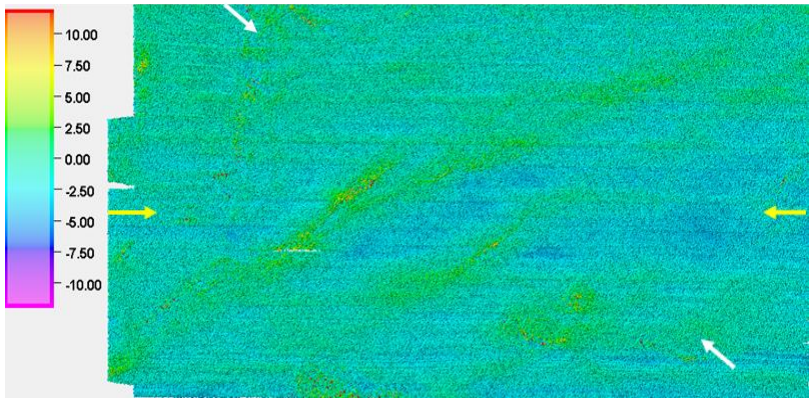

(b)

Figure 5 - water bottom cross-correlation time shift between the Base survey and one underlying survey. The white arrows indicate the shooting direction of the Base survey. The yellow arrows indicate the shooting direction of one underlying survey.

In deep-water environments, ocean currents can mix water of varying salinity and temperature that causes variations of the velocity in the water layer over time. The acquisition of the legacy surveys spanned more than 10 years, and one of the underlying surveys took more than one year to acquire. Considering the ultra-deep water, water velocity correction (WVC), (Carvill, 2009) is critical to align all the data to the same reference water velocity. The reference water velocity is derived according to the TS Dip measurement from the Base survey. Figure 5 shows the water bottom cross-correlation time shift between the Base survey and one of the underlying NAZ surveys. Before WVC, the time shift between the two surveys is more than \pm 12 ms. The time shift variation across the white arrow direction is due to the water velocity variation from the Base survey; while the time shift variation across the yellow arrow direction is related to the water velocity variation of the underlying survey. After WVC, the two surveys are well aligned. The small residual time shifts remaining are reflective of the water bottom topography rather than the shooting direction.

To ensure the alignment of all the events from individual surveys, a statistical wavelet is derived using the near trace stack from each survey. Wavelet processing was conducted to match the amplitude, phase, and spectrum of the underlying surveys to the Base NAZ.

To generate a robust multiple model, all the legacy datasets are integrated in the 3D SRME scheme (Dragoset et al., 2010). Figure 6 demonstrates the required multiple modeling crossline aperture for zero-offset data, by raytracing the velocity model with water bottom, top salt and base salt horizons (Espinoza et al., 2017). The maximum crossline aperture is up to $5 \mathrm{~km}$ due to the highvelocity carbonate in the post salt zone and the steepdipping top salt. The geology-dependent multiple modelling aperture honors the larger aperture where it is needed while saving cost with smaller aperture in other areas. Due to the very limited crossline data coverage of the legacy NAZ data (Table 1), it is not possible to have larger than $1 \mathrm{~km}$ crossline aperture without combining other surveys.

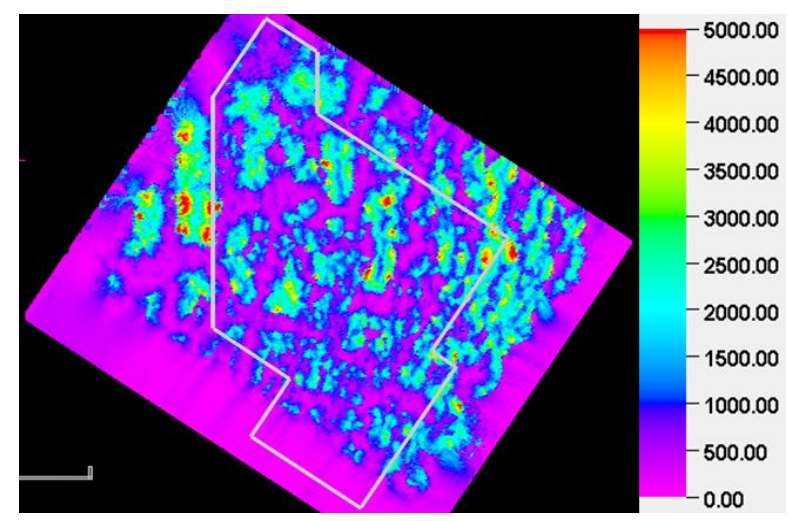

Figure 6 - Crossline aperture map for zero offset data using velocity model raytracing

$45 \mathrm{~Hz} 2 \mathrm{D}$ reverse time migration (RTM) was conducted to demonstrate the effect of MAZ data on SRME (Figure 7). Strong migration swings in Figure $7 a$ are highly suppressed after SRME using constant-aperture multiple model predicted from a single NAZ data (Figure 7b). However, the residual migration swings are still prevalent to interfere with presalt structures. With the improved multiple model using variable aperture and MAZ data, the residual migration swings in Figure $7 \mathrm{~b}$ are very well eliminated (Figure 7c).

Due to the sparse coverage of NAZ 3 data (Table 1), a large percentage of the KDM input data were generated by interpolation using matching-pursuit Fourier interpolation (MPFI), (Schonewille, et. al., 2013) to satisfy $90 \mathrm{~Hz}$ KDM sampling.

\section{Results}


Figure 8 compares a raw shot gather (Figure 8a) after the reprocessing flow (Figure $8 b$ ) and the legacy flow (Figure $8 \mathrm{c})$. The reprocessed data is almost noise free following the improved random and linear noise removal. The seismic events become much more continuous with improved noise attenuation and broadband processing. Following the demultiple, some high-frequency residual surface multiples (Yellow arrows in Figure 8c) exist in the legacy processing, although mostly are hidden by the high amplitude noise. These are addressed in the reprocessing by improved multiple attenuation with geology-dependent variable-aperture SRME (Figure $8 b$ ).

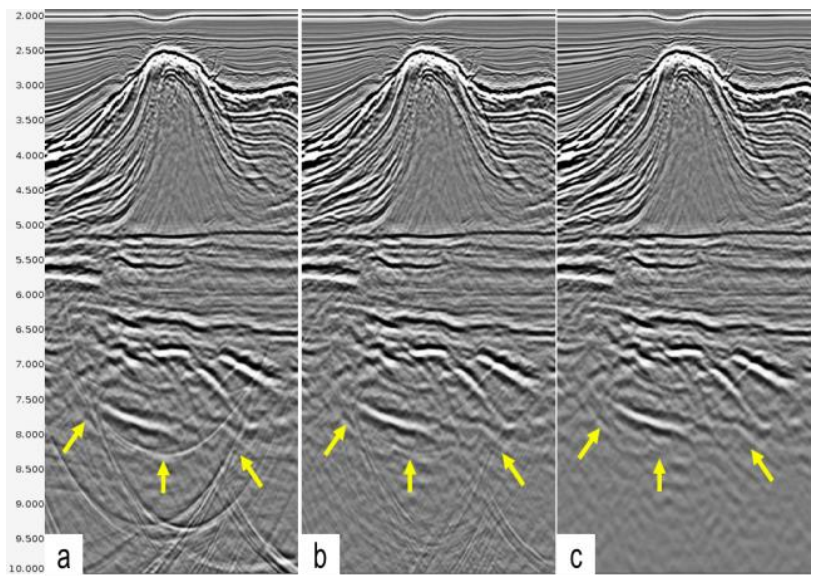

Figure 7- $45 \mathrm{~Hz} 2 \mathrm{D}$ RTM using the data before SRME (a), after SRME using the predicted model from single-azimuth data and constant aperture (b), and after SRME using predicted model from MAZ data and variable aperture (c).

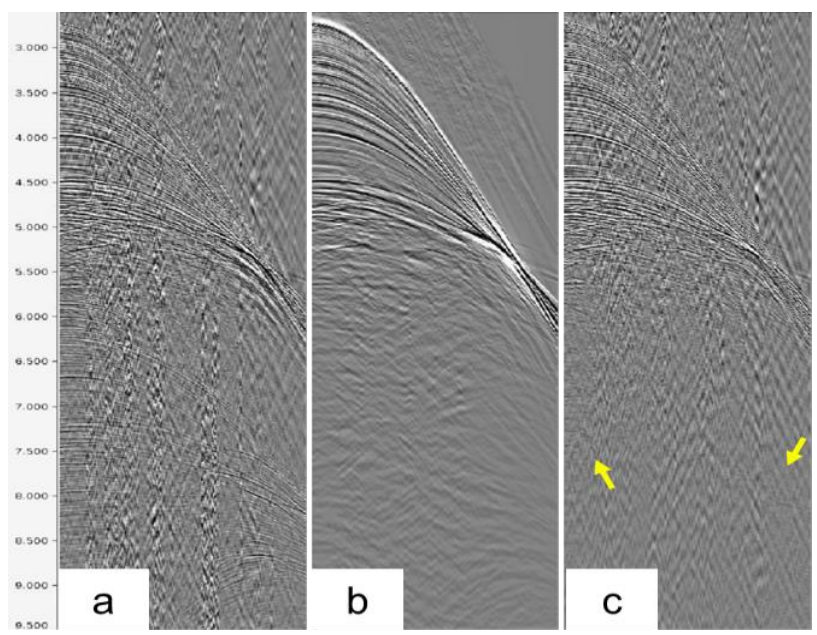

Figure 8 - (a) raw shot gather; (b) final reprocessed shot after ADNA, residual noise attenuation, $A D$, and SRME; (c) 2015 legacy final processed shot. The yellow arrows point some high frequency residual multiples that are behind the strong noise.

To demonstrate the consistency and matching among the different datasets, a KDM common-image-point (CIP) gather is shown for the three NAZ surveys (Figure 9). The red line at $\sim 2.2 \mathrm{~km}$ indicates the depth of the water bottom reflection. The gathers from the three NAZ data align very well with highly similar wavelet characters. All three gathers are quite flat from top to bottom, demonstrating a relatively accurate final velocity model. By comparing the three gathers, the presalt events (Below $5.5 \mathrm{~km}$ ) extend to the farthest offset on the NAZ 2 data. The maximum offset for the NAZ 3 is $6 \mathrm{~km}$, while the other two are $8 \mathrm{~km}$. The coherency variation of the presalt events in the MAZ data indicate that, optimally summing the MAZ data will achieve the premium presalt imaging and angle stacks.

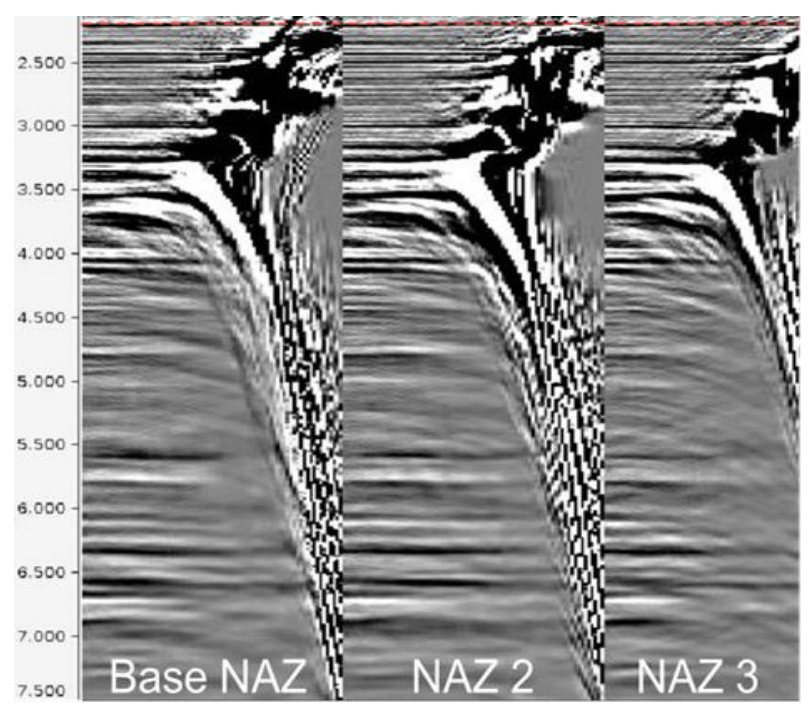

Figure 9 - Central Santos MAZ reimaging KDM CIP gather from the three $N A Z$ surveys.

Figure 10 compares the legacy final enhanced KDM image (10a) with the MAZ final enhanced (10b) KDM image. The SNR is significantly improved in the new image inside salt and in the presalt section. Compared to the non-deghosted legacy data, with broadband processed MAZ data, the top of pure salt (Green arrows) is clearly defined with improved low frequencies; the resolution of the MAZ image is improved with ghost removal. In the legacy image, strong migration swings are observed in the post salt (Pink arrow), inside salt (Red arrows), in the presalt (Dark red arrows), and at the basement (Blue arrow), due to insufficient illumination, a less accurate velocity model, residual surface multiples, and etc. With the improved velocity model and signal processing, the migration swings are mostly suppressed in the MAZ image. The triangle shape base salt (Yellow arrow) is continuously imaged on the MAZ image, but almost invisible on the legacy image, which is close to a well location. The base salt horizon (Orange arrows) is very weak or broken by migration swings on the legacy image, which is almost continuous across the whole reprocessed image. The presalt layers, the faults, and the basement top (Sky blue) are much better defined on the MAZ image. The sharper wavelet in the reprocessing results in high-resolution imaging of the postsalt layers, the small features and the fault zones are clearly delineated (Figure 10c, 10d). 


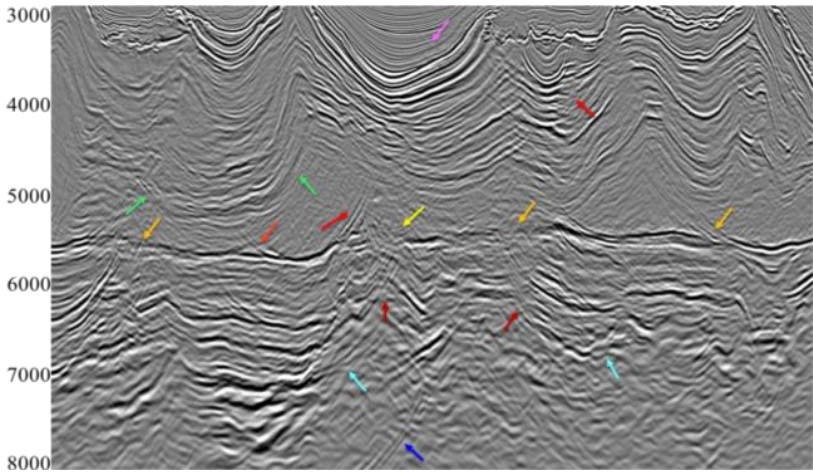

(a)

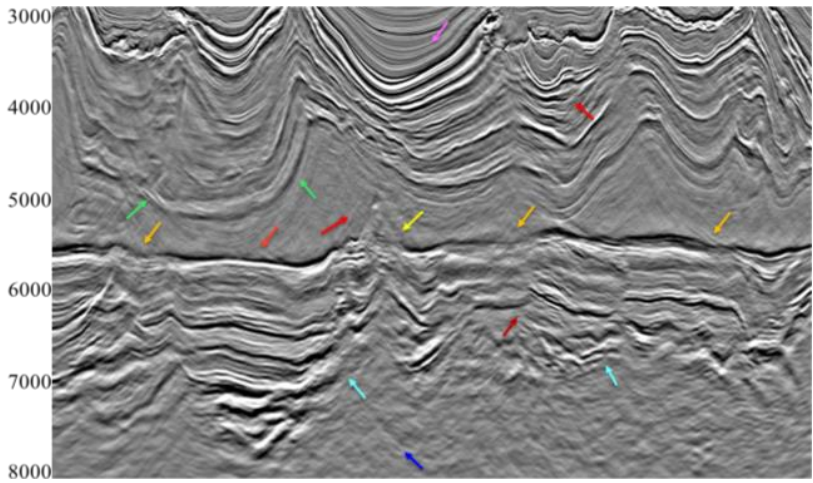

(b)

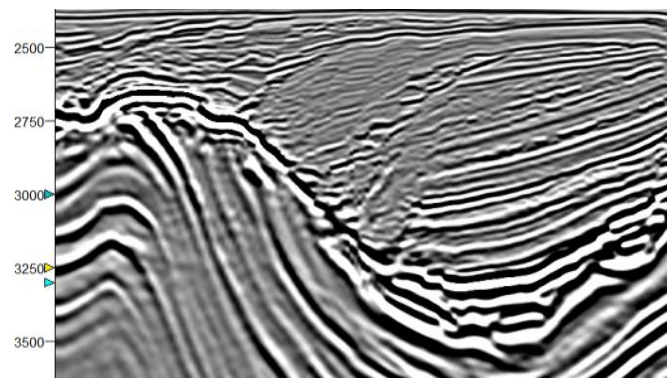

(c)

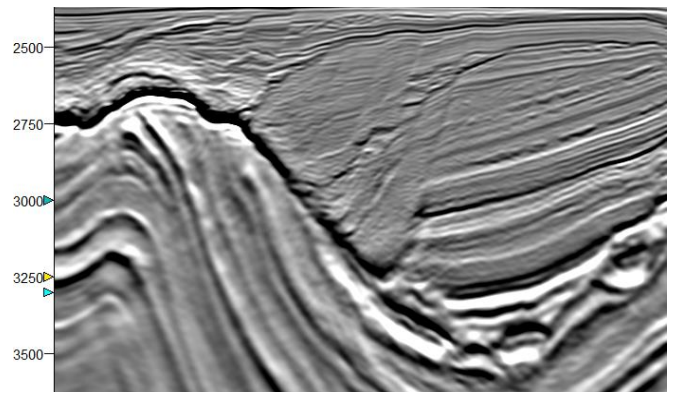

(d)

Figure 10 - (a) the final legacy pre-stack KDM image; (b) the CSMR raw KDM image from the Base survey; (c) the CSMR final enhanced KDM image. (d) and (e) are the zoomed image to focus on the post salt session of (a) and (c). Red arrow - residual surface multiple; green arrow top of pure salt; yellow arrow - migration swings at and below the base salt; yellow arrow - migration swings in the post salt session.

\section{Conclusions}

We presented a contemporary re-processing sequence that successfully applied to the legacy deep-water streamer datasets in the Santos Basin, Brazil. ADNA noise attenuation together with improved residual noise attenuation brings cleaner data for the adaptive deghosting process. The water velocity correction and subsequent wavelet processing ensured the alignment of all the legacy surveys. The highly matched MAZ data make it possible to apply geology-dependent variable aperture to predict multiple model for improved SRME. With the significant added value of MAZ data, in conjunction with the key signal processing and model building technologies, a highresolution depth velocity model was inverted, and therefore delivered enhanced final imaging. The significant improvement of the reprocessing over legacy imaging brings significant valueto the legacy data without the need for additional acquisition.

\section{Acknowledgment}

We thank WesternGeco Multiclient for the permission to use the data, and we thank all the people who involved in processing the project.

\section{References}

Carvill, C.; 2009. A new approach to water velocity estimation and correction. 71st EAGE conference abstracts, Doi: https://doi.org/10.3997/2214-

4609.201400384

Dragoset, B.; Verschuur, E.; Moore, I.; and Bisley, R.; 2010, A perspective on 3D surface-related multiple elimination, GEOPHYSICS Vol 75, Doi: https://doi.org/10.1190/1.3475413

Espinoza, C.; Sanger, W.; Nicholes, D.; Graniel, J.; 2017. Geology-constrained data-driven multiple prediction, SEG Technical Program Expanded Abstracts, https://doi.org/10.1190/segam2017-17789671.1

Ortin, M.; Ysaccis, R.; Li, H.; Barros, P.; 2020. Combining wave paths to improve Brazil's pre-salt image, Central Santos Basin multi-azimuth reimaging, SEG Technical Program

Abstracts, Doi: https://doi.org/10.1190/segam20203427597.1

Rickett, J.; 2014. Successes and challenges in 3D interpolation and deghosting of single-component marinestreamer data, SEG Technical Program Expanded Abstract, Doi: https://doi.org/10.1190/segam2014-1159.1

Schonewille, M.; Yan, Z.; Bayly, M.; Bisley, R.; 2013. Matching pursuit Fourier interpolation using priors derived from a second data set, SEG Technical Program Expanded Abstracts, Doi: https://doi.org/10.1190/segam2013-0956.1

Vigh, D.; Starr, E.W.; 2008. 3D prestack plane-wave, fullwaveform inversion. Geophysics, Vol. 73, https://doi.org/10.1190/1.2952623

Yu, M., and Z. Yan, 2011. Flexible surface multiple attenuation using the curvelet transform, SEG Technical 
Program Expanded Abstracts, Doi:

https://doi.org/10.1190/1.3627922

Zarkhidze, A.; Rickett, J.; Oraghalum, E.; Lansky, C.; Raskopin, A.; Bloor, R.; 2016. Adaptive deghosting for complex geometries in the Gulf of Mexico, SEG Technical
Program
Expanded
Abstract:

https://doi.org/10.1190/segam2016-13868686.1 